\title{
D'yakonov-Perel Spin Relaxation Suppressed by an Applied Magnetic Field
}

\author{
Z. WILAMOWSKI \\ Institute of Physics, Polish Academy of Sciences \\ al. Lotników 32/46, 02-668 Warsaw, Poland
}

AND W. JANTSCH

Institut für Halbleiterphysik, Johannes Kepler Universität, 4040 Linz, Austria

\begin{abstract}
We observe a strong anisotropy of spin relaxation and a decrease in the spin relaxation rate with increasing electron mobility in contrast to predictions of the classical D'yakonov-Perel spin relaxation model. We show that for high electron mobility the cyclotron motion causes an additional modulation of spin-orbit coupling, leading to an effective suppression of the spin relaxation rate.
\end{abstract}

PACS numbers: 72.25.Rb, 73.21.Fg, 85.75.-d

\section{Introduction}

Presently, the concepts of spintronics are developed which involve the utilization of both the electronic- and spin degrees of freedom. Carriers can be easily manipulated by applying external voltages - a well known tool of classical electronics. The fast spin relaxation of conduction electrons usually implies, however, serious limitations for most applications. Therefore, analysis and understanding of the relevant spin relaxation mechanisms and the search for an appropriate material are of a basic importance. The spin relaxation time in III-V compounds is below a nanosecond [1] and under optimal conditions still a fraction of a microsecond. Here silicon, due to much weaker spin-orbit coupling, could be substantially better suited.

In two-dimensional (2D) Si layers in Si/SiGe structures spin relaxation times of the order of a few microseconds have been measured by electron spin resonance 
(ESR) techniques [2-5]. It was shown that the Bychkov-Rashba (BR) spin-orbit coupling [6] is the main origin of spin relaxation in the $2 \mathrm{D}$ electron gas of a modulation doped quantum well with high mobility [5].

\section{Model predictions}

The BR coupling acts on the conduction electron spin, $\boldsymbol{\sigma}$, as an effective magnetic field, $\boldsymbol{B}_{\mathrm{BR}}$. This Rashba field is oriented in-plane of the $2 \mathrm{D}$ layer and perpendicular to electron momentum, $\hbar \boldsymbol{k}$. The resulting $-\boldsymbol{k}$-dependent - zero field splitting is $\hbar \Omega_{\mathrm{BR}}=g \mu_{\mathrm{B}} \boldsymbol{B}_{\mathrm{BR}} \cdot \boldsymbol{\sigma}$.

The direction of the BR field depends on the direction of the $k$-vector of the electron and the spread of $k$-vectors results in a spread of the BR field. Consequently, the ESR frequency is shifted and the ESR line is broadened. The momentum scattering rate, $1 / \tau_{k}$, causes a time modulation of $\mathrm{BR}$ field which leads to the so-called D'yakonov-Perel (DP) spin relaxation [7]. Modulation of the BR field leads to motional narrowing of the spread of BR field. The narrowed line width, i.e., the spin decoherence rate, $1 / T_{2}$, is expected to be proportional to $\tau_{k}$. Also the longitudinal spin relaxation rate, $1 / T_{1}$, corresponding to the spin lifetime, $T_{1}$, is predicted to be proportional to $\tau_{k}$. The DP model well explains the ESR frequency and the line width if the external magnetic field is directed in the sample plane [5]. For electron concentrations of the order of a few times $10^{11} \mathrm{~cm}^{-2}$ the BR field is of the order of $100 \mathrm{Gs}$.

\section{Results and conclusions}

The total line width caused by the BR field, predicted by the DP model, is expected to be isotropic [8]. In spite of that, the line width observed in our samples is highly anisotropic. As an example, the dependence of the ESR line width on the direction of applied field is shown in Fig. 1. For perpendicular orientation of the magnetic field, $\theta=0$, the line width, $\Delta \omega(0)$, is by an order of magnitude smaller as compared to in-plane orientation, $\Delta \omega(90)$. This anisotropy ratio, $\Delta \omega(90) / \Delta \omega(0)$, increases with increasing electron mobility. It is about $\Delta \omega(90) / \Delta \omega(0)=1.5$ for $\tau_{k}^{-1}=10^{12} \mathrm{~s}^{-1}$ and it increases to 10 for $\tau_{k}^{-1}=5 \times 10^{10} \mathrm{~s}^{-1}$.

To explain the observed peculiarities we consider the influence of the cyclotron motion on the spin relaxation. In an external magnetic field, $\boldsymbol{B}$, the cyclotron trajectory is accompanied by a continuously changing BR field. As a consequence, the BR field is additionally modulated leading to a suppression of the spin relaxation by motional narrowing. The cyclotron frequency of $2 \mathrm{D}$ electrons, $\omega_{\mathrm{c}}$, scales with the perpendicular component of the applied field, $B \cos \theta$. Therefore the effective modulation frequency, and thus the resulting spin relaxation rate also depend on $\theta$.

According to general rules, both components of spin relaxation are ruled by the Fourier transforms of the correlation function of the perturbing field [9]. 


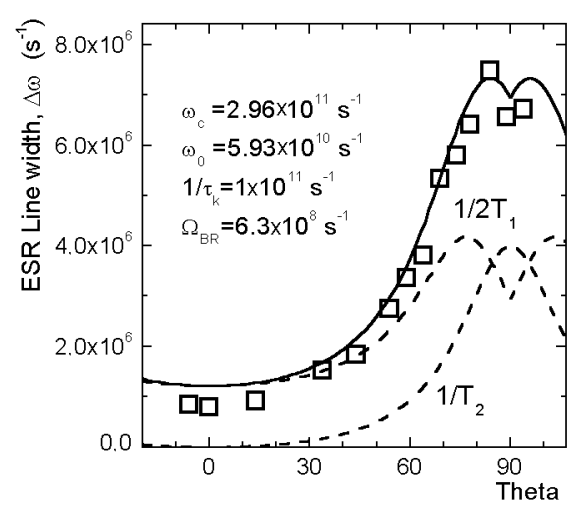

Fig. 1. Dependence of the ESR line width of conduction electrons in a 2D Si/SiGe structure on the direction of applied magnetic field. $\theta=0$ stands for perpendicular direction to the layer. The solid line describing the line width corresponds to Eq. (4), while the transverse and longitudinal components of the spin relaxation rate are marked by dashed lines and described by Eqs. (3), (4).

Momentum scattering and cyclotron motion lead to the following expression for the autocorrelation function of the BR perturbation:

$$
C(\tau)=\left\langle\boldsymbol{\Omega}_{\mathrm{BR}}(\tau), \boldsymbol{\Omega}_{\mathrm{BR}}(0)\right\rangle=\Omega_{\mathrm{BR}}^{2} \exp \left(\mathrm{i} \omega_{\mathrm{c}} \tau-\frac{\tau}{\tau_{k}}\right) .
$$

The resulting expressions for the longitudinal and transverse spin relaxation rates are

$$
\begin{aligned}
\frac{1}{T_{1}} & =\Omega_{\mathrm{BR}}^{2}\left(1+\cos ^{2} \theta\right) \frac{\tau_{k}}{1+\left(\omega_{0}-\omega_{\mathrm{c}}\right)^{2} \tau_{k}^{2}}, \\
\frac{1}{T_{2}} & =\Omega_{\mathrm{BR}}^{2} \frac{\sin ^{2} \theta}{2} \frac{\tau_{k}}{1+\omega_{\mathrm{c}}^{2} \tau_{k}^{2}} .
\end{aligned}
$$

Here $\omega_{0}$ is the Larmor frequency, $\Omega_{\mathrm{BR}}^{2}\left(1+\cos ^{2} \theta\right)$ - the variance of the perpendicular and $\Omega_{\mathrm{BR}}^{2} \sin ^{2} \theta$ - of the longitudinal component of the BR field distribution. The total line width is

$$
\Delta \omega=\frac{1}{2 T_{1}}+\frac{1}{T_{2}}
$$

The lines in Fig. 1 correspond to Eqs. (2)-(4). $\Omega_{\mathrm{BR}}$ is the only fitting parameter. The observed angular dependence is well described by our model according to Eqs. (2)-(4). A characteristic maximum of the line width occurs at $\theta \approx 80^{\circ}$, corresponding to a resonance-like condition at $\omega_{0} \approx \omega_{\mathrm{c}}$, where the energy of the cyclotron motion can be transferred to the spin system. $\Omega_{\mathrm{BR}}$ is the only fitting parameter, but the anisotropy ratio does not depend on $\Omega_{\mathrm{BR}}$. In that sense, the theoretical prediction of a suppression of the spin relaxation, and equivalent to it, of the extraordinarily large anisotropy ratio, is described without any fitting parameter. 
The present model implies also a strong dependence of the suppression of spin relaxation on the electron mobility. For low mobility, $\omega_{\mathrm{c}} \tau_{k} \ll 1$, Eqs. (2) and (3) take the classical form. The DP relaxation rate is expected to be proportional to the momentum relaxation time. The spin relaxation rate thus should increase with increasing mobility. For $\omega_{\mathrm{c}} \tau_{k}>1$, however, the opposite dependence is expected for the spin relaxation rates according to Eqs. (3) and (4): here the higher mobility leads to slower spin relaxation.

\section{Acknowledgment}

We thank F. Schäffler (JKU) for generously providing samples. Work supported within the State Committee for Scientific Research grants PBZ-044/P03/ 2001 and 2 P03B 05423 in Poland and in Austria by the FWF, and ÖAD, both Vienna.

\section{References}

[1] J.M. Kikkawa, D.D. Awshalom, Phys. Rev. Lett. 80, 4313 (1998).

[2] W. Jantsch, Z. Wilamowski, N. Sandersfeld, F. Schäffler, Phys. Status Solidi B 210, 643 (1998).

[3] C.F.O. Graeff, M.S. Brandt, M. Stutzmann, M. Holzmann, G. Abstreiter, F. Schäffler, Phys. Rev. B 59, 13242 (1999).

[4] Z. Wilamowski, N. Sandersfeld, W. Jantsch, D. Töben, F. Schäffler, Phys. Rev. Lett. 87, 026401 (2001).

[5] Z. Wilamowski, W. Jantsch, H. Malisa, U. Rössler, Phys. Rev. B 66, 195315 (2002).

[6] Yu.L. Bychkov, E.I. Rashba, J. Phys. C 17, 6039 (1984).

[7] M.I. D'yakonov, V.I. Perel', Sov. Phys. JETP 38, 177 (1973).

[8] N.S. Avierkiev, L.E. Golub, M. Willander, J. Phys., Condens. Matter 14, R271 (2002).

[9] A. Abragam, Principles of Nuclear Magnetism, Clarendon Press, Oxford 1961. 